\title{
BET Proteins Are Required for Transcriptional Activation of the Senescent Islet Cell Secretome in Type 1 Diabetes
}

\author{
Peter J. Thompson *,+(D, Ajit Shah ${ }^{\dagger}$, Hara Apostolopolou and Anil Bhushan \\ Diabetes Center, University of California San Francisco, 513 Parnassus ave, San Francisco, CA 94143, USA; \\ ajitnshah@icloud.com (A.S.); Hara.apostolopolou@ucsf.edu (H.A.); anil.bhushan@ucsf.edu (A.B.) \\ * Correspondence: pjt2@alumni.ubc.ca \\ † These authors contributed equally.
}

Received: 21 August 2019; Accepted: 24 September 2019; Published: 26 September 2019

\begin{abstract}
Type 1 diabetes (T1D) results from the progressive loss of pancreatic beta cells as a result of autoimmune destruction. We recently reported that during the natural history of T1D in humans and the female nonobese diabetic (NOD) mouse model, beta cells acquire a senescence-associated secretory phenotype (SASP) that is a major driver of disease onset and progression, but the mechanisms that activate SASP in beta cells were not explored. Here, we show that the SASP in islet cells is transcriptionally controlled by Bromodomain ExtraTerminal (BET) proteins, including Bromodomain containing protein 4 (BRD4). A chromatin analysis of key beta cell SASP genes in NOD islets revealed binding of BRD4 at active regulatory regions. BET protein inhibition in NOD islets diminished not only the transcriptional activation and secretion of SASP factors, but also the non-cell autonomous activity. BET protein inhibition also decreased the extent of SASP induction in human islets exposed to DNA damage. The BET protein inhibitor iBET-762 prevented diabetes in NOD mice and also attenuated SASP in islet cells in vivo. Taken together, our findings support a crucial role for BET proteins in the activation of the SASP transcriptional program in islet cells. These studies suggest avenues for preventing T1D by transcriptional inhibition of SASP.
\end{abstract}

Keywords: senescence and SASP; beta cells; type 1 diabetes; BET proteins

\section{Introduction}

Type 1 diabetes (T1D) is a chronic metabolic disease of insulin deficiency caused by an organ-specific autoimmune disorder that leads to progressive loss of pancreatic beta cells. The conventional model for T1D pathogenesis posits a loss of peripheral tolerance, resulting in beta cell-specific autoimmunity of cluster of differentiation 4 positive $\left(\mathrm{CD}^{+}\right)$and $\mathrm{CD} 8^{+} \mathrm{T}$ cells that carry out beta cell destruction with support from innate immune cells [1,2]. While $\mathrm{T}$ cell-mediated beta cell destruction is indeed the main driver of the disease, we recently demonstrated that beta cells actively participate in the process by undergoing DNA damage-induced senescence, upregulation of the pro-survival factor B cell lymphoma 2 (Bcl-2), and acquiring a senescence-associated secretory phenotype (SASP) [3]. Selective ablation of senescent beta cells with small molecule senolytic compounds targeting Bcl-2 prevented T1D in the nonobese diabetic (NOD) mouse model by halting the autoimmune destruction process and preserving beta cell mass [3]. These results demonstrate a causal role for SASP beta cells in the pathogenesis of T1D; however, the mechanisms of SASP activation in beta cells have not been determined.

SASP is controlled at multiple levels, including the level of chromatin and transcription [4,5]. Genes encoding SASP factors are dramatically upregulated in senescent cells, which generally correlates 
with increased protein level and secretion [6,7]. SASP occurs in the context of persistent DNA damage response (DDR) signaling and results from extensive chromatin remodeling and activation of regulatory regions of the genes that encode SASP factors [8-11]. Recent efforts have revealed a growing list of transcription factors, chromatin architectural proteins, and modifiers that collaborate to orchestrate the activation of SASP genes, leading to the development of the secretory phenotype. These include factors such as ATM, macroH2A1, HMGB2, MLL1, BRD4, NF-kB, CEBP $\beta$, Mitf, and PARP-1, and inhibition or depletion of these factors in senescent cells reduces SASP gene activation and subsequent protein secretion $[5,8-10,12,13]$. Activation of SASP genes is also accompanied by changes in chromatin modifications, including the acquisition of marks associated with active enhancers, such as histone H3K27 acetylation (H3K27ac) [8]. Because most of the previous studies investigated the chromatin remodeling and activation of SASP genes in human primary fibroblast lines, it remains unclear whether some of the same transcription factors and chromatin modifiers are required to activate SASP in other cell types and in vivo. Owing to the ability of accumulated SASP cells to profoundly alter tissue microenvironments and disrupt normal processes [14,15], pharmacological approaches to inhibit SASP at the transcriptional level could prove useful for mitigating the effects of senescent beta cells in T1D.

Here, we show that transcriptional activation of the SASP in islets relies upon epigenetic readers of histone lysine acetylation the BET proteins, including BRD4. BRD4 was bound to active SASP gene enhancers marked with H3K27ac in NOD islets. Inhibition of BRD4 chromatin binding with the potent small molecule BET inhibitor iBET-762 led to diminished SASP gene expression, protein secretion and SASP paracrine activities from NOD islets. We further show that BET protein transcriptional activity is required for SASP in human islets undergoing DNA damage. Finally, treatment of NOD mice with iBET-762 prevented diabetes and attenuated SASP in islets in vivo. Taken together, these results demonstrate that BET proteins are key transcriptional activators of SASP in islet cells and indicate that the mechanism of diabetes prevention with BET inhibitors involves attenuation of SASP.

\section{Results}

\subsection{BRD4 Is Required for SASP Gene Activation in NOD Islets}

We previously demonstrated the expression of SASP genes in beta cells at 14-16 weeks in euglycemic NOD mice [3]. To confirm that SASP gene regulatory regions were activated in NOD islets, we performed chromatin immunoprecipitation (ChIP) for H3K27ac. Female NOD mice were used throughout our studies because of their higher disease penetrance [16]. We used the dbSUPER superenhancer database [17] and the VISTA enhancer database (Lawrence Berkeley National Lab) to identify putative enhancers of SASP genes from different mouse cell types. ChIP analysis revealed enrichment of H3K27ac within the enhancer of $M m p 2$ and the promoter/enhancer of $I l 6$ relative to distal regions in the islets of some euglycemic NOD mice, but not others (Figure 1a), consistent with the heterogeneity in the extent of beta cell SASP among mice at the same age [3]. Epigenetic readers of $\mathrm{H} 3 \mathrm{~K} 27 \mathrm{ac}$ include the $\mathrm{BET}$ proteins BRD2, BRD3, and BRD4, which are expressed in most somatic cell types, but are recruited to regulatory regions to activate transcription. BRD4 is a crucial activator of SASP gene enhancers in human fibroblasts [8,18]; therefore, we focused on this paralogue. Immunohistochemistry (IHC) demonstrated the broad expression of BRD4 in beta cells, other non-beta islet cells, and exocrine acinar cells of eight-week-old euglycemic NOD mice (Figure 1b), in which SASP beta cells are rare [3], consistent with the notion that BRD4 expression alone does not induce SASP. To determine whether BRD4 binds to SASP gene regulatory regions in islets, ChIP analysis was performed on islets pooled from multiple NOD mice at 14 weeks of age. This revealed that BRD4 was specifically enriched at the same regulatory regions of Mmp2 and Il6 that harbored H3K27ac, and treatment of islets with the potent small molecule BET inhibitor iBET-762 disrupted its binding at these sites (Figure 1c). To determine whether BRD4 activates SASP genes in specifically in islet cells, dispersed islets isolated from euglycemic 14-week-old NOD mice were depleted of infiltrated $\mathrm{CD} 45^{+}$immune cells to enrich for endocrine islet cells and then incubated with iBET-762 (Figure 1d). 
iBET-762-treated islet cell cultures showed reduced expression of specific beta cell SASP genes Mmp2, Il6, Flnb, and Igfbp4 [3] (Figure 1d). As iBET-762 can affect the chromatin binding of all three BET paralogues, we also used siRNA knockdown to assess the specific contribution of BRD4. siRNA knockdown of Brd4 in NOD islets diminished Il6 expression (Figure 1e). Together, these results implicate BET proteins, and in particular, BRD4, as activators of SASP gene expression in NOD islets.

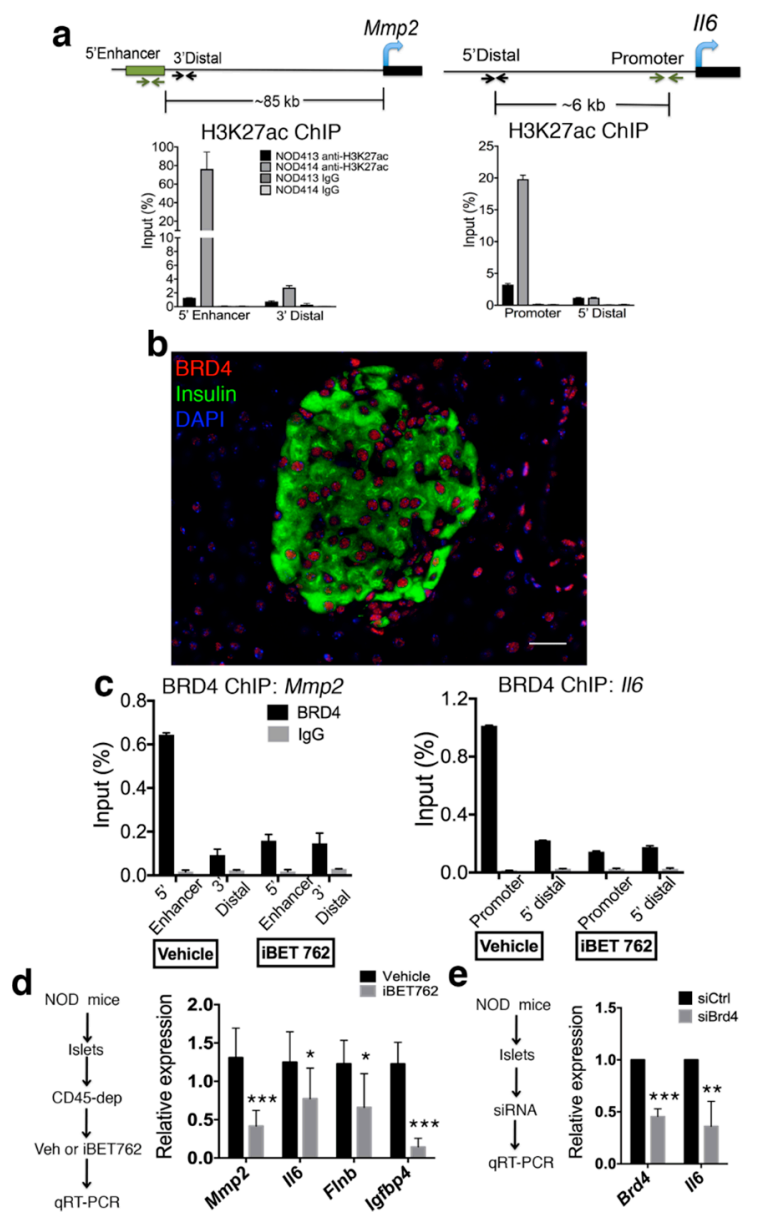

Figure 1. BET protein BRD4 is required for transcriptional activation of senescence-associated secretory phenotype (SASP) genes in nonobese diabetic (NOD) islets. (a) Chromatin immunoprecipitation and quantitative polymerase chain reaction (ChIP and qPCR) for H3K27ac at the enhancer of Mmp2 and promoter of $I l 6$, along with distal negative regions, on islets isolated from two different euglycemic 14-week-old NOD mice (NOD413, NOD414), $n=2$ biological replicates per mouse. Female mice are used throughout our study. The schematic shows the positions of the regions in the ChIP. Immunoglobulin G (IgG) ChIP was a negative control. Error bars are SD. (b) Representative IHC for BRD4 and insulin with DAPI on pancreas section from euglycemic NOD mice at eight weeks of age. Scale bar $=20 \mu \mathrm{m}$. (c) ChIP and qPCR for BRD4 at the same regulatory regions of Mmp2 and Il6 shown in (a) on pooled islets from euglycemic 14-week-old NOD mice cultured for $18 \mathrm{~h}$ with vehicle or iBET-762 ( $n=2$ biological replicates per group), error bars are SD. IgG ChIP was a negative control. (d) Islets isolated from 14-week-old NOD mice were depleted of CD45 ${ }^{+}$immune cells to enrich for endocrine islet cells and then cultured for $24 \mathrm{~h}$ with vehicle (Dimethyl Sulfoxide, DMSO) or iBET-762 followed by quantitative reverse transcription polymerase chain reaction (qRT-PCR) analysis. qRT-PCR of SASP genes Mmp2, $I l 6$, Flnb, and $I g f b p 4$ show the average relative expression for vehicle ( $n=5$ biological replicates) or iBET-762 treated ( $n=4$ biological replicates) islets cells. Error bars are SD. (e) qRT-PCR for Brd4 and Il6 on 14-week-old NOD islets transfected with non-targeting control or Brd4 siRNAs. Data show average expression levels in siBrd4 relative to siCtrl from $n=3$ independent experiments. Error bars are SD. For all panels, ${ }^{*} p<0.05,{ }^{* *} p<0.005,{ }^{* * *} p<0.0005$, two-tailed $t$-tests. 


\subsection{BET Inhibition Blunts SASP Secretion and Paracrine Activities}

Next, we determined whether disrupting BET protein chromatin binding affected SASP protein secretion. To this end, we cultured NOD islets in iBET-762 or vehicle and collected conditioned media (CM) for analysis of SASP factors by luminex assay (Figure 2a). Notably, iBET-762-treated islets showed significantly lower secretion of SASP factors IL-6, Igfbp3, Mmp3, and Mmp12 (Figure 2a). The beta cell SASP exerts non-cell autonomous effects including the ability to induce senescence-related changes in immature islet cells and stimulate the chemotaxis of monocytes in vitro [3]. To address whether iBET-762 treatment affected the paracrine senescence activities of SASP in the CM, we cultured islets from three-week-old NOD mice with CM from islets depleted of CD45 ${ }^{+}$cells of vehicle or iBET-762-treated 14-week-old NOD mice (Figure 2b). While CM from vehicle-treated islets showed induction of $C d k n 1 a$ expression, consistent with paracrine senescence, the iBET-762-treated islet CM did not (Figure 2b). Finally, we tested whether the CM from iBET-treated NOD islets had effects on monocyte chemotaxis using the transwell assay (Boyden chamber assay) on the human THP-1 monocyte cell line. Importantly, CM from NOD islet cells cultured with iBET-762 showed diminished chemotactic activity relative to the vehicle-treated islet CM (Figure 2c), in line with their lower SASP factor secretion (Figure 2a). These data demonstrate that disruption of BET protein transcriptional activity in islets leads to decreased SASP secretion and non-cell autonomous activities.
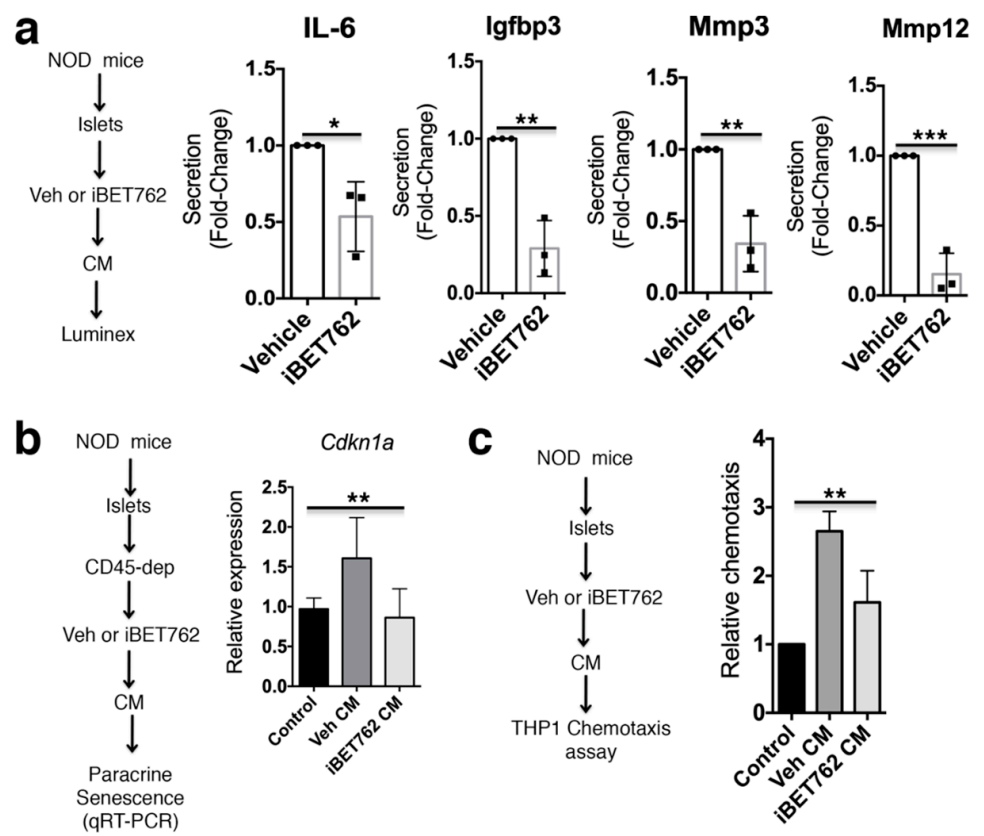

Figure 2. iBET-762 attenuates SASP secretion and paracrine activities from NOD islets. (a) Islets isolated from 14-week-old NOD mice were cultured in vehicle or iBET-762 for $24 \mathrm{~h}$ and the conditioned media (CM) was collected for luminex assays. Relative quantification of average secretion of SASP factors IL-6, Igfbp3, Mmp3, and Mmp12 from iBET-762-treated islets versus vehicle-control islets, $n=3$ biological replicates per group. Error bars are SD. ${ }^{*} p<0.05,{ }^{* *} p<0.005,{ }^{* * *} p<0.0005$, two-tailed $t$-tests. (b) Islets isolated from 14-week-old NOD mice were depleted of CD45 ${ }^{+}$cells and cultured in vehicle or iBET-762 for $24 \mathrm{~h}$ and the resulting CM was collected used in paracrine senescence induction assays on islets from three-week-old old NOD mice, where qRT-PCR for Cdkn1a was a readout of paracrine senescence induction. Regular islet media (not conditioned by islets) was used as a negative control to set the baseline. Data are average relative expression levels from $n=5$ or 6 biological replicates per group, error bars are SD. ${ }^{* *} p<0.005$, one-way analysis of variance (ANOVA). (c) CM was collected from islets of 14-week-old NOD mice cultured with vehicle or iBET-762 and used in chemotaxis assays on THP-1 cells. Regular islet media (not conditioned by islets) was used as a negative control to measure the baseline. Data are chemotaxis fold-change, relative to control, from $n=3$ biological replicates per group. Error bars are SD. ${ }^{* *} p<0.005$, one-way ANOVA. 


\subsection{BET Proteins Are Required for SASP in Human Islets}

We previously showed by IHC that SASP beta cells accumulate in T1D donors as compared with nondiabetic donors [3]. IHC of BRD4 in age-matched human nondiabetic, autoantibody-positive (positive for serum beta cell autoantibody, but not diabetic) and T1D donor pancreas sections showed that it was expressed in the vast majority of $\mathrm{INS}^{+}$and $\mathrm{INS}^{-}$islet cells and acinar cells in each donor (Figure 3a), similar to the staining in 8-week-old NOD mice (Figure 1b). The similar expression patterns in islets of nondiabetic and T1D donors suggest that BRD4 alone is insufficient to induce SASP. Induction of DNA damage in human islets ex vivo can lead to acquisition of an SASP reminiscent of the SASP expressed in beta cells of T1D donors [3]. To determine whether BET proteins are recruited to activate SASP in human islets, we utilized our previous approach for inducing SASP in human islets [3]. Human islets were first treated with bleomycin (bleo) to induce DNA damage or vehicle as a control. Then, after washout of bleo or vehicle, islets were cultured in the presence of vehicle or iBET-762 and monitored for persistent DDR and SASP development by qRT-PCR and luminex assays six days later (Figure 3b). Bleo treatment induced sustained expression of CDKN1A relative to the vehicle control in two different preparations of donor islets (male and female), whereas CDKN2A (INK4A/P16) was unaffected (Figure 3c), consistent with previous our findings [3]. In contrast, iBET-762 treatment diminished the expression CDKN1A after bleo (Figure 3c), suggesting a reduction in the extent of persistent DDR signaling. Furthermore, while bleo-treated islets developed a SASP, as evidenced by upregulated secretion of SASP factors CXCL1, IL-8, and IGFPB4, iBET-762 treatment after bleo attenuated the secretion (Figure 3d). Taken together, these data demonstrate that BET proteins are required for SASP activation in human islets.

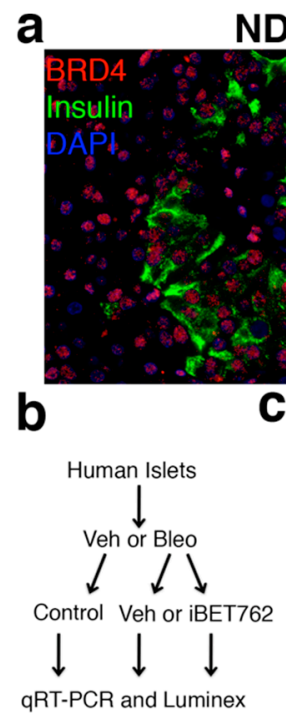

ND

AA+

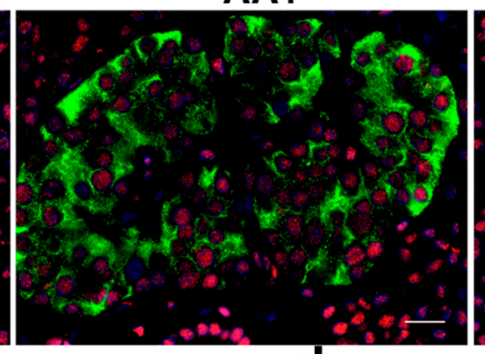

Donor \#2

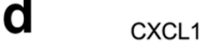

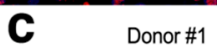

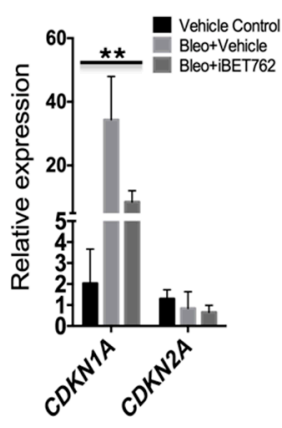

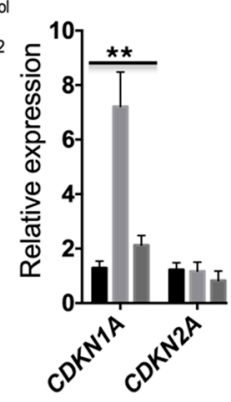
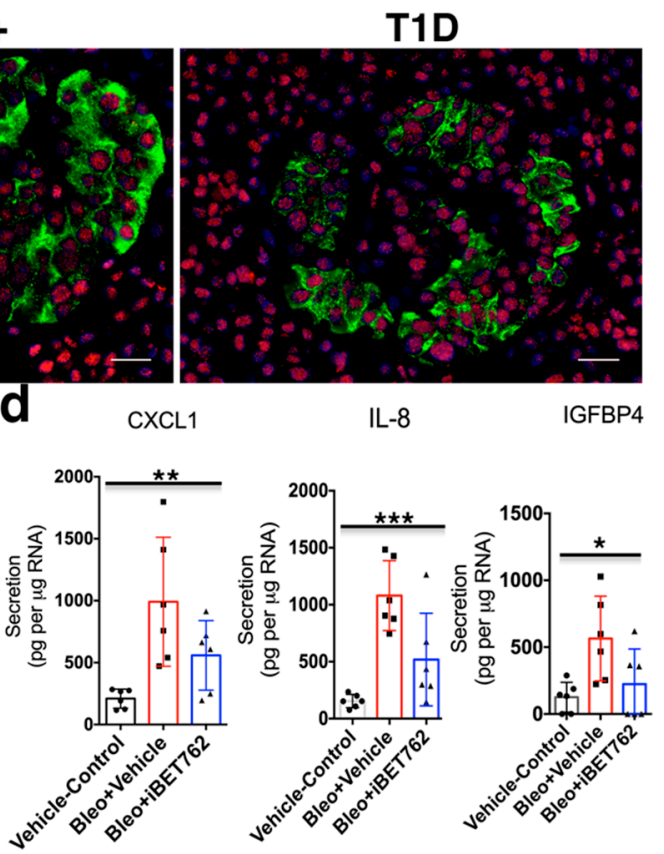

Figure 3. BET proteins are required for activation of SASP in human islets exposed to DNA damage. (a) Representative IHC for BRD4 and Insulin with DAPI in pancreas sections from nondiabetic (ND), autoantibody-positive (AA+), and Type 1 Diabetes (T1D) donors. Scale bars $=20 \mu \mathrm{m}$. (b) Islets from nondiabetic human donors were cultured with bleomycin (bleo) to induce DNA damage, or vehicle $(0.1 \%$ DMSO) as a control, followed by replacing the media with media containing iBET-762 or vehicle and culture for an additional five days. Then, islets were harvested for qRT-PCR and conditioned media (CM) saved for luminex assays. (c) qRT-PCR analysis of CDKN1A and CDKN2A (INK4A/P16) on vehicle control, Bleo + vehicle, or Bleo + iBET-762 islets isolated from two different donors $(n=3$ biological replicates per group for each donor). Error bars are SD. ${ }^{* *} p<0.005$, one-way ANOVA. 
(d) Luminex assay of average protein secretion of SASP factors CXCL1, IL-8, and IGFPB4 from the same islets as in (c). Each dot represents a biological replicate of islets $(n=6$ per group, from the two different donors shown in (c). Error bars are SD. ${ }^{*} p<0.05,{ }^{* *} p<0.005,{ }^{* * *} p<0.0005$, one-way ANOVA.

\subsection{BET Protein Inhibition Diminishes SASP In Vivo}

Previous work has shown that inhibition of BET proteins with the earlier generation inhibitor iBET-151 protects against diabetes in NOD mice [19]. However, the mechanisms of its action on beta cells were not determined. To investigate this mechanism, we first treated a cohort of 12 -week-old euglycemic NOD mice ( $n=10$ mice per group) with either vehicle or iBET-762 over a two-week period, and then monitored random blood glucose levels weekly until 30 weeks of age (Figure 4a). Blood glucose measurements showed that $60 \%$ of vehicle-injected mice developed diabetes by 30 weeks of age, whereas only $10 \%$ of the iBET-762 injected mice developed hyperglycemia by 30 weeks of age $(p=0.0115$, Figure 4a). Histological staining of pancreas sections confirmed that a greater number of islets was preserved intact in iBET-762-treated mice (Figure 4b), indicating that iBET-762 halted disease progression, consistent with the previous study using iBET-151 [19].

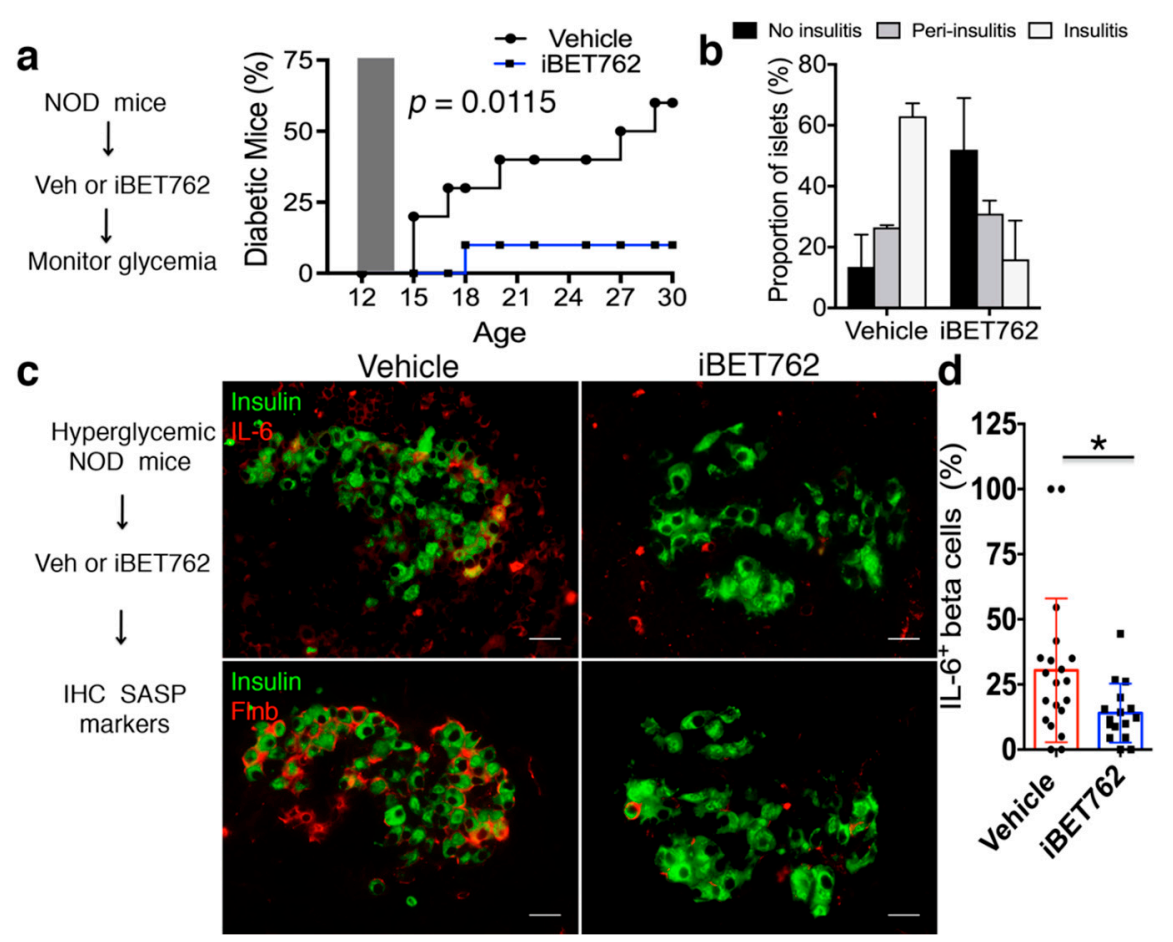

Figure 4. iBET-762 prevents diabetes and attenuates SASP in vivo. (a) Twelve-week-old NOD mice ( $n=10$ per group) were administered vehicle or iBET-762 intraperitoneally (i.p.) for two weeks and glycemia was monitored up to 30 weeks of age. Diabetes incidence curves show the frequency of hyperglycemic mice at each time point. Gray shaded area shows treatment window (12-14 weeks of age). $p=0.0115$, log-rank test. (b) Insulits grading in $n=30$ islets taken from two euglycemic vehicle control and two euglycemic iBET-762 mice after the prevention study in (a) at 30 weeks. Error bars are SD. (c) Recent onset hyperglycemic NOD mice were administered vehicle or iBET-762 i.p. for one week and then IHC was performed for SASP markers. Representative IHC of SASP factors IL-6 or Flnb with insulin in pancreas sections from the indicated diabetic mice after treatment. Scale bars $=$ $20 \mu \mathrm{m}$. (d) Quantification of IL- $6^{+} / \mathrm{Ins}^{+}$cells per islet in the hyperglycemic NOD mice treated as in (a). Each dot represents an islet, where $n=20$ islets for a total of 735 beta cells were scored from three vehicle-treated mice, and $n=16$ islets for a total of 891 beta cells were scored from three iBET-762-treated mice. ${ }^{*} p<0.05$, two-tailed $t$-test. 
We hypothesized that the protective effect of BET protein inhibition resulted at least in part from its inhibition of SASP. Consistent with this, treatment of recent onset hyperglycemic NOD mice with iBET-151 also had a beneficial effect on beta cell function [19], which would be expected if this treatment affects the SASP program. To test whether BET inhibition diminished the SASP in vivo, recent onset hyperglycemic NOD mice were injected every day for one week with either vehicle or iBET-762 and pancreas sections were immunostained for SASP markers (Figure 4c). Vehicle-injected hyperglycemic NOD mice showed a robust expression of IL-6 and Flnb in Ins ${ }^{+}$cells and in some Ins ${ }^{-}$ islet cells (Figure 4c). In contrast, islets from iBET-762-injected hyperglycemic mice showed fewer cells expressing IL-6 or Flnb (Figure 4c). The frequency of IL- $6^{+} / \mathrm{Ins}^{+}$(beta) cells per islet was variable in hyperglycemic mice, similar to our previous observations of SASP heterogeneity in beta cells of euglycemic NOD mice [3]. Nevertheless, quantifications showed an approximately $50 \%$ reduction in the average frequency of IL- $6^{+}$beta cells per islet in iBET-762-treated hyperglycemic mice relative to vehicle controls ( $30 \%$ in vehicle versus $14 \%$ in iBET-762, Figure $4 \mathrm{~d}$ ). Taken together, these results demonstrate that BET inhibition with iBET-762, which is sufficient to prevent diabetes in NOD mice, also blunts SASP in vivo.

\section{Discussion}

Our findings implicate BET proteins as crucial transcriptional activators of SASP in islets cells of mice and humans. Our findings also indicate that in NOD mice, the protection afforded by BET inhibitors against diabetes involves attenuation of SASP. However, it is important to recognize that because BET proteins are also expressed in some immune cell types, where they activate genes for immune responses, BET inhibitors can also block immune inflammatory responses unrelated to SASP [18]. In addition, like their predecessor JQ1, second-generation BET inhibitors such as iBET-151 and the more potent derivative iBET-762 affect the chromatin binding of all of the paralogues [20], and there may be different contributions of BRD2, BRD3, and BRD4 to SASP activation in islets versus inflammatory responses in immune cells. Regardless, the net-effect of BET inhibition in the NOD model is remarkably beneficial, perhaps as a result of both diminishing SASP and reducing inflammatory gene expression in immune cells [19].

Transcriptional inhibition of SASP genes with iBET-762 in NOD islets had a corresponding downstream effect on SASP factor secretion and paracrine activity. Remarkably, BET inhibition also affected the extent of persistent DDR and SASP in human islets exposed to DNA damage, revealing a conservation of this mechanism in human islets. Inhibition of BRD4 in human fibroblasts after the induction of senescence also inhibits activation of SASP [8], consistent with our findings in human islets. While we focused on BRD4 in the current study, genetic approaches will be required to delineate contributions of BRD2 and BRD3 to SASP activation in mouse and human islets.

The mechanism by which BET proteins are recruited to SASP gene regulatory regions in islet cells remains to be determined. BRD4 was expressed ubiquitously throughout islets and in acinar cells in NOD mice and humans, indicating that it is not sufficient to drive SASP activation on its own. BRD4 interacts with NF- $\mathrm{KB}$ to facilitate transcriptional activation of SASP genes in fibroblasts $[8,21]$. Although this pathway may also operate during SASP in islets, there may also be islet cell-specific transcription factors induced during senescence that recruit BET protein activity. Islet cell-specific transcription factors that interact with BRD4 would be attractive targets for designing small molecules to selectively inhibit SASP gene expression. Unlike BET inhibitors, this approach would disentangle the effects of SASP inhibition in islet cells from the inhibitory effects on inflammatory responses in immune cells.

Selective targeting of transcriptional and epigenetic regulators such as the BET protein family using small molecule probes is emerging as a powerful approach to treat a variety of diseases resulting from their aberrant activity [20]. As BET proteins contribute to the SASP transcriptional program in human islets, our work suggests that this concept may also be relevant to the prevention and/or 
treatment of T1D in humans. In conclusion, targeted inhibition of the SASP transcriptional program may provide a new opportunity to develop therapies for T1D.

\section{Materials and Methods}

\subsection{Animal Husbandry and Procedures}

All procedures involving mice were performed according to the IACUC standards following ethics approval by the animal committee at the University of California San Francisco (AN159774-02A, 1 September 2019). Female NOD/ShiLtJ mice (from Jackson Labs) or NOD/MrkTac (from Taconic Labs) were purchased at eight weeks of age and allowed to age further in our colony. Mice were housed under standard conditions with a $12 \mathrm{~h} \mathrm{light/dark} \mathrm{cycle.} \mathrm{To} \mathrm{measure} \mathrm{glycemia,} 1 \mathrm{~mm}$ of tail was clipped and blood was tested with a glucometer and disposable test strips (Freestyle Lite, Alameda, CA USA). Mice were considered hyperglycemic with a single blood glucose measurement of $>150 \mathrm{mg} / \mathrm{dL}$ for fasting blood glucose (FBG) or two consecutive measurements on different days of $>300 \mathrm{mg} / \mathrm{dL}$ for random blood glucose (RBG). For the diabetes prevention study, euglycemic NOD/MrkTac mice $(n=20)$ at 12 weeks of age were split into two groups of 10 , where one group was injected i.p. with iBET-762 (30 mg/kg) dissolved in DMSO as stock solution of $50 \mathrm{mg} / \mathrm{mL}$, which was further diluted in saline to a concentration of $5 \mathrm{mg} / \mathrm{mL}$. For the control group, an equivalent volume of vehicle $(10 \%$ DMSO in saline) solution was injected. For iBET and vehicle groups, the total volume injected was between 150 and $200 \mu \mathrm{L}$. The treatment was carried out for 14 consecutive days. Following this, both groups of mice were monitored for RBG. The RBG was measured once per week until they reached 30 weeks of age. At the end of the study, pancreata from the remaining euglycemic mice were harvested, formaldehyde-fixed, and paraffin-embedded. For assessment of insulitis, $5 \mu \mathrm{m}$ paraffin-embedded sections from two vehicle and two iBET762-injected mice at 30 weeks of age from regions $50 \mu \mathrm{m}$ apart in the pancreas block were stained with hematoxylin and eosin. Insulitis was scored for three different stages based on the level of infiltration of islet by immune cells, no insulitis, peri-insulitis (infiltration at the periphery of the islets), and insulitis (infiltration within the islet) with $n=30$ islets were scored per mouse. For the iBET treatment of hyperglycemic mice, once the NOD/MrkTac were hyperglycemic, as defined by RBG $>300 \mathrm{mg} / \mathrm{dL}$, they were injected i.p. with iBET-762 $(30 \mathrm{mg} / \mathrm{kg})$ or vehicle for seven consecutive days, and then sacrificed for pancreas dissection and histology.

\subsection{Human Pancreas Sections and Islets}

Human pancreas sections were obtained from the Network for Pancreatic Organ donors with Diabetes (nPOD) program [22]. nPOD pancreas donors used in this study were nPOD Case IDs: 6279 (nondiabetic, 19-year-old male), 6397 (Glutamic acid decarboxylase autoantibody (GADA) positive 21-year-old female), 6342 (Islet tyrosine phosphatase 2 autoantibody (IA-2A), Microinsulin autoantibody (mIAA) double-positive, T1D 14-year-old female). Human islets were obtained from the Integrated Islet Distribution Program (IIDP). Human islet preparations used in this study were as follows: Donor \#1: RRID:SAMN10784981, 45-year-old female, body mass index (BMI) = 35.7 and Donor \#2: RRID:SAMN11046361, 57-year-old male, BMI = 35.9.

\subsection{Culture of Mouse Islets, Inhibitors and RNAi}

Islets from NOD/ShiLt mice were isolated according to standard methods by collagenase digestion through the bile duct. For collection of conditioned media (CM) from whole islets, after $1-2 \mathrm{~h}$ of resting at $37^{\circ} \mathrm{C}$, NOD islets from $n=3$ mice were separated into two equal pools per mouse, where one half was cultured in vehicle $(0.1 \%$ DMSO) and the other half was cultured in $5 \mu \mathrm{M}$ iBET-762 (ApexBio, Houston, TX, USA) in non-treated 96-well plates at $37^{\circ} \mathrm{C}$ with $5 \% \mathrm{CO}_{2}$ in $200 \mu \mathrm{L}$ of serum-free mouse islet media (RPMI 1640, 2 mM L-glutamine, 1X penicillin-streptomycin) for $24 \mathrm{~h}$. Following the incubation, CM was collected for analysis and islet DNA was either extracted immediately (Zymogen total genomic DNA kit, Irvine, CA, USA) or islets were stored at $-80^{\circ} \mathrm{C}$ in genomic DNA lysis buffer to be extracted 
later. Collection of CM from CD45-depleted islet cell cultures was performed as previously [3], where islets were pooled from five or ten 14-week-old NOD mice, depleted of $\mathrm{CD} 45^{+}$immune cells using metal-assisted cell sorting (BioLegend, San Diego, CA, USA) and plated at 60,000-100,000 cells per well (equal numbers per well) in three biological replicates per group in mouse serum-free islet media containing vehicle $(0.1 \%$ DMSO) or $5 \mu \mathrm{M}$ iBET-762 for $24 \mathrm{~h}$. Following incubation, the $\mathrm{CM}$ was collected for paracrine assays. For qRT-PCR of CD45-depleted islet cultures, cells were incubated with iBET-762 in media containing 10\% Fetal Bovine Serum (FBS) (rather than in serum-free media). For RNAi, after recovery of NOD islets for $18 \mathrm{~h}$ in serum-containing islet media (RPMI 1640, $2 \mathrm{mM}$ L-glutamine, 1X penicillin-streptomycin, 10\% FBS), 20 islets per well ( $n=3$ biological replicates per group) were transfected with control (non-targeting \#2) siRNA or mouse Brd4 SMARTpool siRNA (Dharmacon GE Life Sciences, Lafeyette, CO, USA) at $100 \mathrm{nM}$ each with lipofectamine 3000 according to the product instructions. Islets were cultured for an additional $72 \mathrm{~h}$ and then harvested for RNA extraction. THP-1 human monocytic leukemia cells (purchased from ATCC, Gaithersburg, MD, USA) were cultured in RPMI-1640, $10 \%$ FBS, and $1 \mathrm{X}$ penicillin-streptomycin at $37^{\circ} \mathrm{C}$ with $5 \% \mathrm{CO}_{2}$.

\subsection{Culture of Human Islets and Inhibitors}

Human islets from IIDP were cultured as previously [3] in RPMI-1640 containing 10\% FBS, $5.5 \mathrm{mM}$ glucose, and $1 \mathrm{X}$ antibiotic-antimycotic (Gibco, Grand Island, NY, USA). Islets were rested for $24 \mathrm{~h}$ in culture prior to inducing DNA damage with $50 \mu \mathrm{M}$ bleomycin. Forty-eight hours later, bleomycin was removed and fresh media was added, and islets were then cultured with vehicle ( $0.1 \%$ DMSO) or $5 \mu \mathrm{M}$ iBET-762. Five days later, islets were transferred into serum-free media for the collection of conditioned media (CM). After $24 \mathrm{~h}, \mathrm{CM}$ was collected for luminex assays and islets were harvested for RNA extraction and qRT-PCR.

\subsection{Immunohistochemistry and Quantification}

Immunohistochemistry of formaldehyde-fixed paraffin-embedded pancreas sections was performed as described [23]. Five micron tissue sections were rehydrated with xylene and graded ethanol washes, soaked in $1 \% \mathrm{H}_{2} \mathrm{O}_{2}$ for $10 \mathrm{~min}$, and subjected to heat-mediated antigen retrieval with sodium citrate $\mathrm{pH} 6.0$ for $7.5 \mathrm{~min}$ at $100 \%$ and $14 \mathrm{~min}$ at $50 \%$ power in a $1250 \mathrm{~W}$ microwave. Sections were cooled to room temperature with tap water and then permeabilized with TBS containing $0.1 \%$ Triton-x-100 for five min. Sections were blocked with $2 \%$ normal donkey serum in protein block buffer (Dako) for $15 \mathrm{~min}$ and then incubated overnight at $4{ }^{\circ} \mathrm{C}$ with primary antibodies diluted in DAKO antibody diluent: anti-insulin (DAKO, 1:1000), anti-BRD4 (Bethyl laboratories, 1:10,000, Montgomery, TX, USA), anti-IL-6 (Novus Biologicals 1:300, Centennial, CO, USA), and anti-Flnb (Novus Biologicals, 1:500, Centennial, CO, USA) [3]. Fluorescein isothiocyanate (FITC) or Cy3-conjugated secondary antibodies (Jackson Immunoresearch, West Grove, PA, USA) were used to detect primary antibodies and sections were counterstained with Vectashield with DAPI (Vector Labs, Burlingame, CA, USA). Images were taken on a Zeiss Axioscope2 wide-field fluorescence microscope (Carl Zeiss Vision Inc, San Diego, CA, USA) with Axiovision software. IL-6 was quantified in beta cells from recent onset diabetic NOD mice ranging in ages from 12 to 19 weeks that were vehicle treated $(n=3)$ or iBET-762 treated $(n=3)$, with three pairs of age-matched mice among the groups (12-14 weeks, 16 weeks, 17-19 weeks). Because the mice were diabetic in this study, they had widely varying numbers of remaining islets and remaining beta cells per islet. To account for these differences, frequencies of IL- $6^{+}$beta cells were calculated from a similar number of total beta cells scored from the mice. A total of $n=20$ islets with 735 beta cells was scored from vehicle mice, and $n=16$ islets with 891 beta cells were scored in iBET-762 mice, taking sections throughout the pancreas from mice in both groups. Residual islets containing fewer than 10 beta cells were not scored. 


\subsection{THP-1 Chemotaxis Assay}

Chemotaxis assays were performed as previously described using transwell inserts with $5 \mu \mathrm{m}$ polycarbonate membranes [3]. Briefly, $1 \times 10^{5} \mathrm{THP}-1$ cells were seeded into $150 \mu \mathrm{L}$ of control media (serum-free THP1 media: RPMI 1640, 2 mM L-glutamine, 1\% penicillin-streptomycin) in the upper chamber and $150 \mu \mathrm{L}$ of control media alone or islet $\mathrm{CM}$ (with vehicle or iBET-762 treatment) was added to the lower chamber. Cells were allowed to migrate for $5-6 \mathrm{~h}$ at $37^{\circ} \mathrm{C}$, and then the inserts were removed and the cells in the lower chamber were counted. The control media in the lower chamber was used to determine background levels of migration and the fold-change relative to the control media was used to determine relative chemotaxis in the other samples.

\subsection{Paracrine Senescence Assays}

Paracrine senescence assays were performed as previously described [3]. NOD islets isolated from three-week old mice were cultured for four days with CM from islets of 14 to 15 weeks NOD mice depleted of $\mathrm{CD}_{4} 5^{+}$cells that were treated with vehicle or iBET-762. After culture period the three-week NOD islets were harvested for RNA and qRT-PCR.

\subsection{ChIP and $q P C R$}

Crosslinked ChIP was performed with the MicroChIP kit (Diagenode, Denville, NJ, USA) according to the product instructions. For H3K27ac ChIP, the islets were prepared from 13-14-week-old NOD/MrkTac or NOD/ShiLtJ mice and rested $18 \mathrm{~h}$ in mouse islet media. The following day, all the islets from one mouse each were dissociated into single cell suspension using a nonenzymatic buffer reagent (Gibco) and counted with trypan blue. Approximately $2-5 \times 10^{5}$ cells were crosslinked with $1 \%$ formaldehyde for $10 \mathrm{~min}$ at room temperature, quenched with glycine, and then sonicated with the Diagenode Bioruptor to generate $\sim 200-600$ bp fragments. ChIP was performed with $1 \mu \mathrm{g}$ of anti-H3K27ac (Abcam ab4729, Cambridge, MA, USA) or total rabbit IgG (Sigma-Aldrich, St. Louis, $\mathrm{MO}$, USA) and incubated overnight at $4{ }^{\circ} \mathrm{C}$, followed by capture on protein A dynabeads. Crosslinked ChIP of BRD4 from NOD mouse islets was performed using the True MicroChIP kit (Diagenode) and the same procedure was followed for preparing islets. Then, instead of resting overnight, the islets from five NOD mice were divided into two pools, where one set was incubated with vehicle $(0.1 \%$ DMSO) and the other incubated with $5 \mu \mathrm{M}$ iBET-762 for $18 \mathrm{~h}(n=2$ biological replicates per group). Islets were then dissociated, crosslinked, and sonicated as described above. ChIP was performed using $1 \mu \mathrm{g}$ of anti-BRD4 (Bethyl labs A301-985) or total rabbit IgG (Sigma-Aldrich). Ten percent input fractions from all were saved and DNA extracted in parallel with the ChIP samples for qPCR normalization. qPCR using SYBR green was performed using the following forward and reverse primers (5'-3'): Mmp2 enhancer AACATGCAAGGGAGATCAGC and 5'GGGCTATGAAATGCCAGAAA; Mmp2 distal GCTGGTCCCACAGTGAAGTT and GGACACAGCGCAACTGAATA; Il6 promoter TGTGGGATTTTCCCATGA and TGCCTTCACTTACTTGCAGAGA; Il6 distal GAGAGAGGCAAGCACAGAAA and CCGAGCTGGTTGGTGATAAG. ChIP enrichment was calculated using the percent input method.

\subsection{Quantitative Reverse-Transcription PCR}

Total RNA was extracted from mouse or human islets cultured as described above and treated with DNAse I with the Zymogen RNA extraction micro kit (Zymogen, Irvine, CA, USA) according to the product instructions. RNA was converted into cDNA using oligo(dT) primer and the SuperScript IV kit (Invitrogen, Carlsbad, CA, USA) or the qScript kit (QuantaBio, Beverly, MA, USA). qPCR was performed with the ABI 7900HT system and SDS 2.4 software and target gene primers used previously, and relative quantification was performed with the $\Delta \Delta C_{T}$ method using $R Q$ manager 1.2.1, and expression values were normalized to mouse Ppia or human PPIA as a housekeeping gene [3]. Primer sequences for mouse Il6; Ppia; and human CDKN1A, CDKN2A (p16), and PPIA were as previously described [3]. 
Newly designed forward and reverse primer sequences used in this study were as follows (forward and reverse, $\left.5^{\prime}-3^{\prime}\right)$ : Flnb: AACCAGAACTGGAAGATGG and ATGGCTTCTCGGGCATTATC; Mmp2: CCCCGATCTACACCTACACC and GGAGTGACAGGTCCCAGTGT; Igfbp 4: ATCGAAGCCATCCAGGAAAG and CAGGGGTTGAAGCTGTTGTT; and Brd4: TTCAGCACCTCACTTCGACC and CTGGTGTTTTTGGCTCCTGC.

\subsection{Luminex Assays}

Luminex assays were performed on $50 \mu \mathrm{L}$ of islet $\mathrm{CM}$, according to the product instructions using either the custom human panel (R\&D systems, Minneapolis, MN, USA) reported previously [3] or using a custom mouse panel (R\&D systems) including the following: IL-6, Mmp12, Mmp3, and Igfbp3. Analyte concentrations were determined from standard curves of purified proteins in each assay provided in the kits. For mouse islet secretion assays involving inhibitor treatments, islets were saved for DNA extraction to normalize concentrations to DNA content and data were pg of secreted factor per $\mu \mathrm{g}$ DNA and expressed as a fold-change relative to the pool of vehicle-treated islets from the same mouse ( $n=3$ mice). For human islet secretion assays, data were normalized to RNA content and reported as pg secreted factor per $\mu \mathrm{g}$ RNA.

\subsection{Statistics}

The number of biological replicates, number of mice used, and repeated experiments are indicated in the figure legends. Statistical analyses were performed where experiments were carried out using three or more biological replicates or independent experiments using unpaired one-way ANOVA for groups of three or with unpaired two-tailed $t$-tests for groups of two. Log-rank test was used to compare diabetes incidence curves. The results were considered significant at $p<0.05$. Statistics were computed using GraphPad prism v6.0.

Author Contributions: Conceptualization P.J.T., A.S., and A.B.; methodology, P.J.T., A.S., and A.B.; investigation and experiments, P.J.T., A.S., and H.A.; resources, A.B.; data curation P.J.T. and A.S.; writing-original draft preparation, P.J.T.; writing-review and editing, P.J.T., A.S., and A.B.; visualization, P.J.T.; supervision, A.B.; project administration, A.B.; funding acquisition, P.J.T. and A.B.

Funding: P.J.T. was funded by a grant from the Diabetes Research Connection and a postdoctoral fellowship from the Hillblom Foundation. This research was funded by start-up funds from the UCSF Diabetes Center to A.B.

Acknowledgments: We would like to thank Mark Atkinson for assistance with human donor pancreas specimens through the nPOD repository.

Conflicts of Interest: The authors declare no conflict of interest. The funders had no role in the design of the study; in the collection, analyses, or interpretation of data; in the writing of the manuscript; or in the decision to publish the results.

\section{Abbreviations}

$\begin{array}{ll}\text { BET } & \text { Bromodomain ExtraTerminal domain } \\ \text { NOD } & \text { Nonobese diabetic } \\ \text { qRT-PCR } & \text { Quantitative reverse transcription polymerase chain reaction } \\ \text { ChIP } & \text { Chromatin immunoprecipitation } \\ \text { DMSO } & \text { Dimethyl sulfoxide } \\ \text { DDR } & \text { DNA damage response } \\ \text { SASP } & \text { Senescence-associated secretory phenotype } \\ \text { ATM } & \text { Ataxia Telangiectasia mutated } \\ \text { NF- } k \text { B } & \text { Nuclear Factor kappa B } \\ \text { PARP1 } & \text { Poly-(ADP) ribose polymerase 1 } \\ \text { macroH2A1 } & \text { Macro Histone H2A1 } \\ \text { Bcl-2 } & \text { B cell lymphoma 2 } \\ \text { HMGB2 } & \text { High mobility group protein B2 } \\ \text { CEBP } \beta & \text { CAAT enhancer binding protein beta } \\ \text { MLL1 } & \text { Mixed lineage leukemia 1 }\end{array}$




$\begin{array}{ll}\text { Mitf } & \text { Melanocyte inducing transcription factor } \\ \text { H3K27ac } & \text { Histone H3 Lysine 27 acetylation } \\ \text { dbSUPER } & \text { Database of Superenhancers } \\ \text { BRD4 } & \text { Bromodomain-containing protein 4 } \\ \text { siRNA } & \text { Small interfering RNA } \\ \text { IL-6 } & \text { Interleukin 6 } \\ \text { Flnb } & \text { Filamin B } \\ \text { Mmp2 } & \text { Matrix metalloprotease 2 } \\ \text { Igfbp4 } & \text { Insulin-like growth factor binding protein 4 } \\ \text { Ppia } & \text { Peptidyl prolyl isomerase A } \\ \text { Cdkn1a } & \text { Cyclin dependent kinase inhibitor 1A } \\ \text { Cdkn2a } & \text { Cyclin dependent kinase inhibitor 2A } \\ \text { CD45 } & \text { Cluster of differentiation 45 } \\ \text { FITC } & \text { Fluorescein isothiocyanate } \\ \text { T1D } & \text { Type 1 Diabetes } \\ \text { Cxcl1 } & \text { CXC Chemokine ligand 1 } \\ \text { IL-8 } & \text { Interleukin 8 }\end{array}$

\section{References}

1. Bluestone, J.A.; Herold, K.; Eisenbarth, G. Genetics, pathogenesis and clinical interventions in type 1 diabetes. Nature 2010, 464, 1293-1300. [CrossRef]

2. Christoffersson, G.; Rodriguez-Calvo, T.; von Herrath, M. Recent advances in understanding Type 1 Diabetes. F1000Research 2016, 5, 1-8. [CrossRef] [PubMed]

3. Thompson, P.J.; Shah, A.; Ntranos, V.; Van Gool, F.; Atkinson, M.; Bhushan, A. Targeted Elimination of Senescent Beta Cells Prevents Type 1 Diabetes. Cell Metab. 2019, 29, 1045-1060. [CrossRef] [PubMed]

4. Coppé, J.-P.; Patil, C.K.; Rodier, F.; Sun, Y.; Muñoz, D.P.; Goldstein, J.; Nelson, P.S.; Desprez, P.-Y.; Campisi, J. Senescence-associated secretory phenotypes reveal cell-nonautonomous functions of oncogenic RAS and the p53 tumor suppressor. PLoS Biol. 2008, 6, 2853-2868. [CrossRef] [PubMed]

5. Kuilman, T.; Michaloglou, C.; Vredeveld, L.C.W.; Douma, S.; van Doorn, R.; Desmet, C.J.; Aarden, L.A.; Mooi, W.J.; Peeper, D.S. Oncogene-Induced Senescence Relayed by an Interleukin-Dependent Inflammatory Network. Cell 2008, 133, 1019-1031. [CrossRef] [PubMed]

6. Özcan, S.; Alessio, N.; Acar, M.B.; Mert, E.; Omerli, F.; Peluso, G.; Galderisi, U. Unbiased analysis of senescence associated secretory phenotype (SASP) to identify common components following different genotoxic stresses. Aging (Albany. NY). 2016, 8, 1316-1329. [CrossRef] [PubMed]

7. Freund, A.; Patil, C.K.; Campisi, J. p38MAPK is a novel DNA damage response-independent regulator of the senescence-associated secretory phenotype. EMBO J. 2011, 30, 1536-1548. [CrossRef]

8. Tasdemir, N.; Banito, A.; Roe, J.-S.; Alonso-Curbelo, D.; Camiolo, M.; Tschaharganeh, D.F.; Huang, C.-H.; Aksoy, O.; Bolden, J.E.; Chen, C.-C.; et al. BRD4 Connects Enhancer Remodeling to Senescence Immune Surveillance. Cancer Discov. 2016, 6, 612-629. [CrossRef]

9. Capell, B.C.; Drake, A.M.; Zhu, J.; Shah, P.P.; Dou, Z.; Dorsey, J.; Simola, D.F.; Donahue, G.; Sammons, M.; Rai, T.S.; et al. MLL1 is essential for the senescence- associated secretory phenotype. Genes Dev. 2016, 30, 321-336. [CrossRef]

10. Aird, K.M.; Iwasaki, O.; Kossenkov, A.V.; Tanizawa, H.; Fatkhutdinov, N.; Bitler, B.G.; Le, L.; Alicea, G.; Yang, T.L.; Johnson, F.B.; et al. HMGB2 orchestrates the chromatin landscape of senescence-associated secretory phenotype gene loci. J. Cell Biol. 2016, 215, 325-334. [CrossRef]

11. Rodier, F.; Coppé, J.P.; Patil, C.K.; Hoeijmakers, W.A.M.; Muñoz, D.P.; Raza, S.R.; Freund, A.; Campeau, E.; Davalos, A.R.; Campisi, J. Persistent DNA damage signalling triggers senescence-associated inflammatory cytokine secretion. Nat. Cell Biol. 2009, 11, 973-979. [CrossRef] [PubMed]

12. Chen, H.; Ruiz, P.D.; McKimpson, W.M.; Novikov, L.; Kitsis, R.N.; Gamble, M.J. MacroH2A1 and ATM Play Opposing Roles in Paracrine Senescence and the Senescence-Associated Secretory Phenotype. Mol. Cell 2015, 59, 719-731. [CrossRef] [PubMed] 
13. Ohanna, M.; Giuliano, S.; Bonet, C.; Imbert, V.; Hofman, V.; Zangari, J.; Bille, K.; Robert, C.; Bressac-de Paillerets, B.; Hofman, P.; et al. Senescent cells develop a PARP-1 and nuclear factor- $\mathrm{k}$ B-associated secretome (PNAS). Genes Dev. 2011, 25, 1245-1261. [CrossRef] [PubMed]

14. Kirkland, J.L.; Tchkonia, T. Cellular Senescence: A Translational Perspective. EBioMedicine 2017, $21,21-28$. [CrossRef] [PubMed]

15. Sharpless, N.E.; Sherr, C.J. Forging a signature of in vivo senescence. Nat. Rev. Cancer 2015, 15, $397-408$. [CrossRef] [PubMed]

16. Pearson, J.A.; Wong, F.S.; Wen, L. The importance of the Non Obese Diabetic (NOD) mouse model in autoimmune diabetes. J. Autoimmun. 2016, 66, 76-88. [CrossRef] [PubMed]

17. Khan, A.; Zhang, X. DbSUPER: A database of Super-enhancers in mouse and human genome. Nucleic Acids Res. 2016, 44, D164-D171. [CrossRef]

18. Nicodeme, E.; Jeffrey, K.L.; Schaefer, U.; Beinke, S.; Dewell, S.; Chung, C.; Chandwani, R.; Marazzi, I.; Wilson, P.; Coste, H.; et al. Suppression of inflammation by a synthetic histone mimic. Nature 2010, 468, 1119-1123. [CrossRef]

19. Fu, W.; Farache, J.; Clardy, S.M.; Hattori, K.; Mander, P.; Lee, K.; Rioja, I.; Weissleder, R.; Prinjha, R.K.; Benoist, C.; et al. Epigenetic modulation of type-1 diabetes via a dual effect on pancreatic macrophages and Beta cells. Elife 2014, 3, e04631. [CrossRef]

20. Ferri, E.; Petosa, C.; McKenna, C.E. Bromodomains: Structure, function and pharmacology of inhibition. Biochem. Pharmacol. 2016, 106, 1-18. [CrossRef]

21. Malaquin, N.; Martinez, A.; Rodier, F. Keeping the senescence secretome under control: Molecular reins on the senescence-associated secretory phenotype. Exp. Gerontol. 2016, 82, 39-49. [CrossRef] [PubMed]

22. Campbell-Thompson, M. Organ donor specimens: What can they tell us about type 1 diabetes? Pediatr. Diabetes 2015, 16, 320-330. [CrossRef] [PubMed]

23. Dhawan, S.; Tschen, S.; Zeng, C.; Guo, T.; Hebrok, M.; Matveyenko, A.; Bhushan, A. DNA methylation directs functional maturation of pancreatic $\beta$ cells. J. Clin. Investig. 2015, 125, 2851-2860. [CrossRef] [PubMed]

(C) 2019 by the authors. Licensee MDPI, Basel, Switzerland. This article is an open access article distributed under the terms and conditions of the Creative Commons Attribution (CC BY) license (http://creativecommons.org/licenses/by/4.0/). 